\title{
Imaging and tracking single plasmonic nanoparticles in 3D background-free with four-wave mixing interferometry
}

\author{
P. Borri, G. Zoriniants, N. Giannakopoulou, F. Masia*, I. Pope, and W. Langbein* \\ Cardiff University School of Biosciences, Museum Avenue, Cardiff CF10 3AX, United Kingdom \\ * Cardiff University School of Physics and Astronomy, The Parade, Cardiff CF24 3AA, United Kingdom \\ e-mail: borrip@cf.ac.uk
}

\begin{abstract}
Imaging and tracking single nanoparticles using optical microscopy are powerful techniques with many applications in biology, chemistry, and material sciences. Despite significant advances, imaging single particles in a scattering environment as well as localizing objects with nanometric position precision remains challenging. Applied methods to achieve contrast are dominantly fluorescence based, with fundamental limits in the emitted photon fluxes arising from the excited-state lifetime as well as photobleaching. Here, we show a new four-wave mixing interferometry technique, whereby single nonfluorescing gold nanoparticles are imaged background-free even inside biological cells, and their position determined with nanometric precision in 3D. The technique is also uniquely sensitive to particle asymmetries of only $0.5 \%$ ellipticity, corresponding to a single atomic layer of gold, as well as particle orientation. This method opens new ways of unravelling single-particle trafficking within complex 3D architectures.
\end{abstract}

Keywords: nonlinear optics, multiphoton microscopy, single particle tracking, plasmonics.

\section{INTRODUCTION}

The precise and rapid localization of nano-objects in 3D is essential to identify diffusion mechanisms in complex systems at the molecular level. Especially for biological applications, it is necessary to develop single particle tracking (SPT) technologies capable of high localisation precision in scattering environments in order to unmask the dynamics of proteins, molecular complexes, vesicles, or viruses directly inside living biological systems. To achieve sufficient contrast and specificity against backgrounds in heterogeneous environments, current methods often rely on tracking fluorescing molecules. However, fluorophores are single quantum emitters and thus only capable of emitting a certain maximum number of photons per unit time due to the finite duration of their excited-state lifetime. Moreover, they are prone to photobleaching and associated photo-toxicity. As a result, the spatial position precision and trajectory time window of single particle tracking by means of fluorescent reporters is typically restricted to $20-50 \mathrm{~nm}$ and a few seconds, respectively [1].

Alternatively to using fluorescent emitters, single particle tracking can be implemented with non-fluorescing metallic nanoparticles (NPs) that exhibit strong light scattering and absorption at the localised surface plasmon resonance (LSPR). This signal is photostable, and the achievable photon fluxes are governed by the incident photon fluxes and the NP optical extinction cross-section. Owing to the large number of photons making up the image of an individual particle, its center position coordinates can be determined with high precision, much better than the diffraction limit of a microscope objective. This has been shown using wide-field imaging techniques such as bright-field microscopy [2], dark-field microscopy [3], differential interference contrast (DIC) [4], and interferometric scattering microscopy [5]. These techniques, however, are not background-free and either use large NPs to distinguish them against endogenous scattering and phase contrast in heterogeneous samples or work in optically clear environments. A more selective technique uses photothermal imaging where the image contrast originates from the refractive index change in the region surrounding the nanoparticle due to local heating following light absorption. This is a focused-beam scanning technique and has been used to track single $5 \mathrm{~nm}$ diameter gold NPs in two dimensions (however not 3D) with an estimated $20 \mathrm{~nm}$ precision, using a beam triangulation method at $30 \mathrm{~Hz}$ tracking rate [6]. More recently, single 40nm diameter gold NPs have been imaged in $3 \mathrm{D}$ by photothermal optical coherence microscopy with $0.5 \mu \mathrm{m}$ lateral and $2 \mu \mathrm{m}$ axial resolution, but tracking was not reported [7]. By detecting the nanoparticle only indirectly via the photothermal index change generated in its surrounding, this method is also not free from backgrounds. In fact, photothermal contrast has been shown in the absence of NPs due to endogeneous absorption in cells [8]. Generally, for all these techniques, rapid tracking of small NPs in 3D with high precision in a scattering environment is challenging.

In 2009, we invented, patented and demonstrated four-wave mixing (FWM) imaging interferometry, triplyresonant to the LSPR, as a very selective, high-contrast, photostable method to detect single small $(<40 \mathrm{~nm})$ gold NPs [9]. Here, we present our latest generation of the technique comprising a new FWM detection modality that can determine the position of a single gold NP in the 15-30-nm radius range with shot-noise limited precision better than $20 \mathrm{~nm}$ in plane and $1 \mathrm{~nm}$ axially from scanless single-point background-free acquisition on a $1 \mathrm{~ms}$ time scale, by exploiting optical vortices of tightly focused light. The technique is also uniquely sensitive to particle asymmetries down to $0.5 \%$ ellipticity, corresponding to a single atomic layer of gold. 


\section{FOUR-WAVE MIXING IMAGING}

FWM excitation and detection in our technique can be understood as a pump-probe scheme [10,11], see sketch in Fig.1. The resonant absorption of a 100fs-short pump pulse of intensity $E_{1} E_{1} *$ induces the formation of a hot electron gas in the metal which changes the NP dielectric constant. The corresponding change in the NP transmission or scattering is resonantly probed by a second pulse of field $\mathrm{E}_{2}$ at an adjustable delay time $\tau$ after

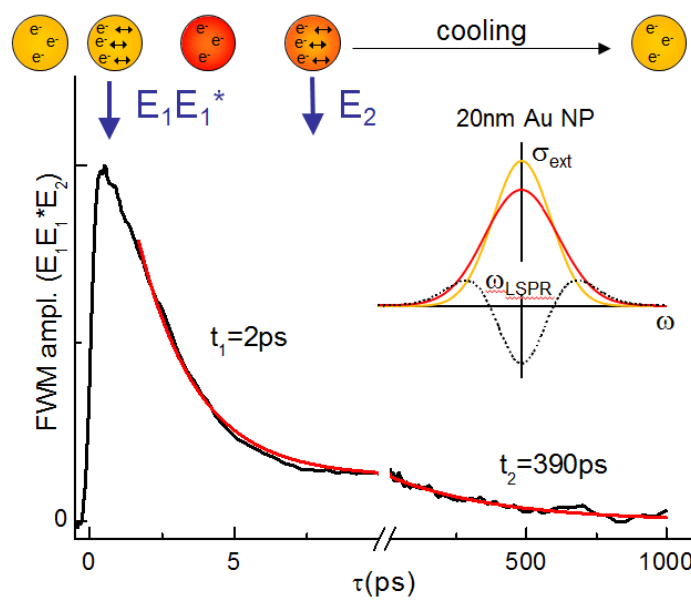

Fig.1. Pump-probe FWM detection of a single gold NP (see text). The inset sketches the LSPR extinction spectrum for a cold (yellow) and hot (red) electron plasma. the pump-pulse, resulting in a FWM field proportional to $\mathrm{E}_{1} \mathrm{E}_{1} * \mathrm{E}_{2}$. For detection, the pump intensity is modulated at frequency $v_{\mathrm{m}}(0.4 \mathrm{MHz})$, the frequency of $\mathrm{E}_{2}$ is shifted by $v_{2}$ $(82 \mathrm{MHz})$, and the FWM field interferes with an un-shifted reference pulse. This enables heterodyne detection of the FWM field at the corresponding beat radiofrequency $v_{2}-v_{L}$ $\pm v_{\mathrm{m}}\left(v_{\mathrm{L}}=80 \mathrm{MHz}\right.$ is the laser pulse repetition rate) separated from $E_{1}$ and $E_{2}$ and thus free from scattering background. Notably, the interferometric detection is also insensitive to incoherent fluorescence backgrounds. By varying $\tau$, the electron dynamics can be time-resolved, revealing the time scale of the thermalisation between electron and the lattice of the metal ( 1 ps) and the subsequent thermalisation of the NP with the surrounding medium (>100 ps). This dynamics enables us to distinguish the electronic NP response from instantaneous coherent backgrounds and/or long-lived photothermal effects. As a result, we can achieve completely background-free FWM imaging of single gold NPs even in scattering and fluorescing cellular environments.

To exemplify this point, Fig. 2 shows fixed HeLa cells that have internalized gold NPs of $20 \mathrm{~nm}$ radius, imaged with FWM using a 1.45 NA oil-immersion objective. High-resolution DIC microscopy was available in the same instrument. Furthermore, the set-up enables simultaneous interferometric detection of the reflected probe field (for details see [12]). Figure 2(a) shows the DIC image of a group of HeLa cells on which reflection

Single Au NPs in HeLa cells:

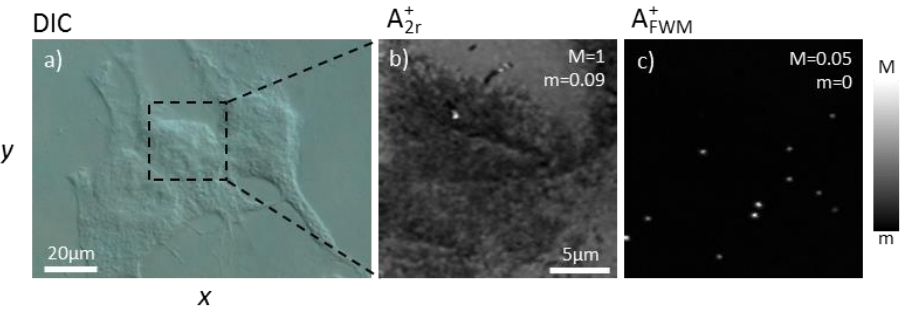

Fig.2. Background-free FWM imaging of single $40 \mathrm{~nm}$ gold NPs in HeLa cells (c). The heterogeneity of the cell is shown with differential interference contrast $(a)$ and reflectometry $(b)$. FWM was acquired with a pump-probe delay time of $0.5 \mathrm{ps}$, pump (probe) power at the sample of $30 \mu \mathrm{W}(15 \mu \mathrm{W}), 2 \mathrm{~ms}$ pixel dwell time. and FWM imaging was performed in the region highlighted by the dashed frame. The reflection image (amplitude of the reflected probe field) shown in Fig. 2(b) correlates with the cell contour seen in DIC and shows a spatially varying contrast due to thickness and refractive index inhomogeneities in the sample. Even with a particle diameter as large as $40 \mathrm{~nm}$, gold NPs are not distinguished from the cellular contrast in DIC or in the reflection image. On the contrary, the FWM field amplitude shown in Fig.2(c) as a maximum intensity projection over a $6 \mu \mathrm{m} \quad \mathrm{z}$ stack is background-free (throughout the $\mathrm{z}$ stack) and clearly indicates the location of single gold NPs in the cell.

\section{FOUR-WAVE MIXING SINGLE PARTICLE TRACKING}

Compared to our previous works [9-11], here we have introduced two significant improvements to the set-up. These are a) reflection (epi) collection geometry (as opposed to transmission) and b) dual circular-polarisation detection. Modification a) enables us to localise the axial position of a single gold NP with single-point acquisition in the $1 \mathrm{~ms}$ range at an estimated $0.5 \mathrm{~nm}$ shot-noise limited precision (see Fig.3). This is because in the epi-geometry a change in the particle axial position results in a change of the optical path length of the reflected wave. The coherent interferometric detection of the phase $\left(\Phi^{+}{ }_{\text {FWM }}\right)$ of the reflected FWM field thus directly encodes the particle axial coordinate (which is not possible in transmission geometry). Modification b) allows for the detection of the particle in-plane coordinates (see Fig.4). Due to the high numerical aperture (NA) of the microscope objective and the vectorial nature of the light field, there is a significant cross-circularly polarised field component that forms an optical vortex of $l=2$ topological charge in the focal plane (i.e. it has an amplitude which is zero in the focus centre, increases proportional to the square of the radius away from the 


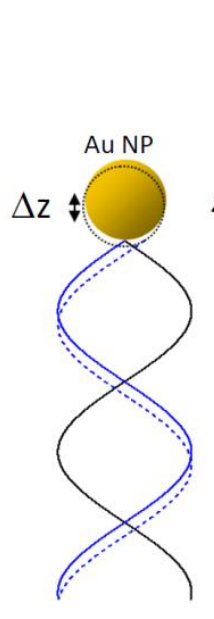

$\Delta \Phi \propto \Delta z$
Calculations:

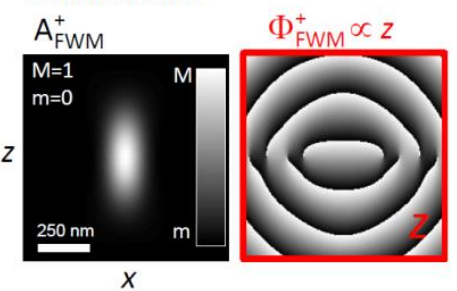

Experiments:

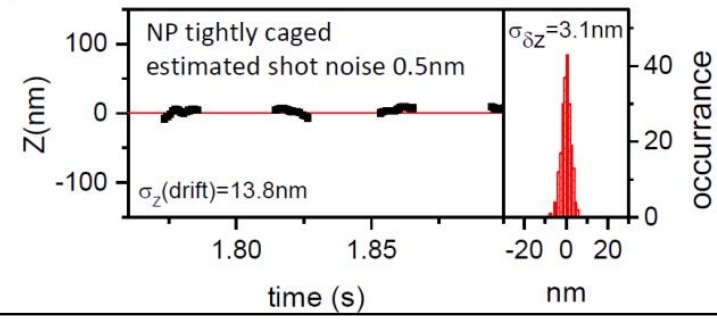

Fig.3. Axial $(z)$ localisation via the phase of the reflected FWM field. Photon shot-noise precision at $1 \mathrm{~ms}$ acquisition on a $50 \mathrm{~nm}$ diameter gold NP is estimated to be $0.5 \mathrm{~nm}$. Measured localisation is practically limited by noise in the sample stage $(3 \mathrm{~nm} \mathrm{rms})$ and drifts (for more details see also [12]).
NP in agarose pocket:

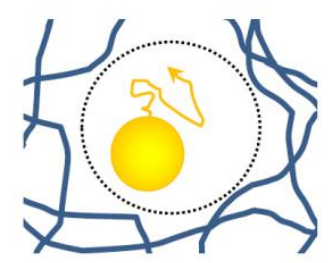

centre, and a phase changing with twice the in-plane polar angle). Owing to the presence of such an optical vortex field pattern, an off-centre inplane particle position results in a non-zero FWM field component cross-circularly polarised (relative to the input probe circular polarisation), with an amplitude $\left(\mathrm{A}_{\mathrm{FWM}}^{-}\right)$and phase $\left(\Phi_{\mathrm{FWM}}^{-}\right)$measured at a single point (i.e. scanless) which encode the direction and position of the particle.

Notably, we found that a tiny deviation from perfect spherical shape of the NP in-plane results in a crosscircularly polarised component. This was evident when measuring gold NPs immobilised onto a glass surface. We were able to model our experimental findings by assuming and ellipsoid nanoparticle in the dipole limit, and in turn calculating the pump-induced change of the particle polarizability probed by $\mathrm{E}_{2}$ and interferometrically detected through its interference with a mode-matched reference field, as a function of the NP position in the sample focal plane (for more details see [12]). These calculations, and their comparison with experiments on a nominally spherical $30 \mathrm{~nm}$ radius NP are shown in Fig.4. From the calculations, we find that the non-zero value of $A_{F W M}^{-}$in the focus centre scales linearly with the particle ellipticity in-plane, and that the value of $\Phi_{\text {FWM }}^{-}$in the focus depends on the particle orientation angle. We can explain the experimental results shown in Fig.4, by assuming NP semi-axes of $\mathrm{a}=30.135 \mathrm{~nm}$ and $\mathrm{b}=\mathrm{c}=30 \mathrm{~nm}$ along the $\mathrm{x}, \mathrm{y}$, and $\mathrm{z}$ axes in the particle reference system, and the longer axis being rotated in plane relative to the $\mathrm{x}$-axis in laboratory system by about 150 degrees. It is remarkable that an asymmetry of only $0.5 \%$ in aspect ratio, or about one atomic layer of gold, manifests as a significant perturbation of the cross-polarized field patterns compared to the spherical case.

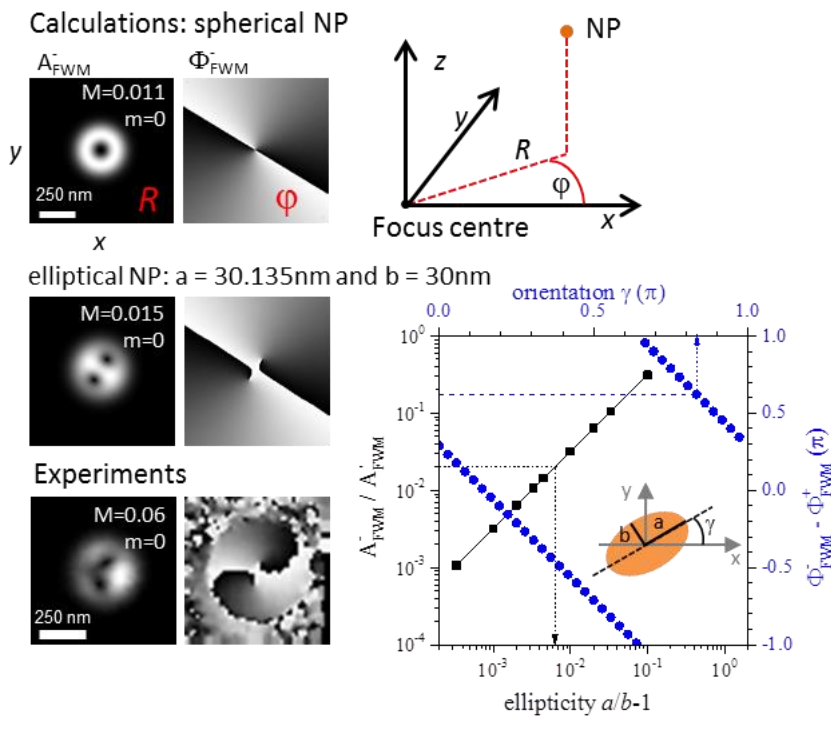

Fig.4. Top: Concept of in-plane localisation with $l=2$ optical vortex in the cross-circularly polarised FWM field, for a 1.45 NA objective. Bottom: Experiments and calculations of the effect of NP asymmetry (see text).
When NPs are not immobilized onto a surface but are freely rotating (a more relevant scenario for particle-tracking applications), we can expect that the acquired field components time averaged over a sufficiently long acquisition time would no longer be affected by the particle asymmetry since this is averaged upon rotation. For this purpose we investigated single gold NPs of $25 \mathrm{~nm}$ radius embedded in a dense $(5 \% \mathrm{w} / \mathrm{v})$ agarose gel in water and measured using an index-matched 1.27 NA water-immersion objective. Specifically, we looked at NPs that were freely rotating but enclosed in a tight pocket of the agar network, resulting in a negligible average translation during the measurement time. In this case, we were able to demonstrate that the NP position retrieval based on our FWM read-out resulted in a localisation with $16 \mathrm{~nm}$ precision in plane and 3 $\mathrm{nm}$ axially from rapid single-point measurements at $1 \mathrm{~ms}$ acquisition time. The precision in plane is consistent with the estimated photon shot-noise, while axially it is limited by the nano-positioning sample stage (see Fig.3, and [12]). 


\section{CONCLUSIONS}

We have shown our latest generation nonlinear microscopy technique based on the interferometric detection of the polarization-resolved resonant FWM field in amplitude and phase from a single gold nanoparticle, with a high numerical aperture objective. FWM detection is completely background-free in scattering environments such as biological cells and it outperforms existing methods such as reflectometry, scattering, and differential interference contrast. Notably, the technique provides a new method to determine the position of a single nonfluorescing gold NP with nanometric precision in three dimensions from scanless far-field optical measurements. The displacement of the nanoparticle from the center focus in the axial direction is directly determined from the epi-detected FWM field phase, while the in-plane displacement manifests as a crosscircularly polarized component because of the optical vortex field pattern in the focus of a high numerical aperture objective, the amplitude and phase of which enables accurate position retrieval.

Shape asymmetry of the NP of as little as $0.5 \%$ ellipticity, corresponding to about one atomic layer of gold, also induces a cross-circularly polarized field. This exceptional sensitivity to asymmetry eventually limits accurate position retrieval in-plane. This can be overcome by observing a NP freely rotating, such that rotational averaging of the asymmetry occurs over the acquisition time.

\section{ACKNOWLEDGEMENTS}

This work was funded by the UK EPSRC Research Council (Grants No. EP/I005072/1, No. EP/I016260/1, No. EP/L001470/1, No. EP/J021334/1, and No. EP/M028313/1) and by the EU (FP7 Grant No. ITN FINON 607842). P. B. acknowledges the Royal Society for funding (grant no. WM140077). We acknowledge Lukas Payne for help in sample preparation and TEM characterization, and Peter Watson, Paul Moody, and Arwyn Jones for help in cell culture protocols.

\section{REFERENCES}

[1] C. Manzo and M. F. Garcia-Parajo: A Review of Progress in Single Particle Tracking: From Methods to Biophysical Insights, Rep. Prog. Phys. 78, 124601, 2015.

[2] T. Fujiwara et al.: Phospholipids Undergo Hop Diffusion in Compartmentalized Cell Membrane, J. Cell Biol. 157, 1071 (2002).

[3] H. Ueno, et al:: Simple Dark-Field Microscopy with Nanometer Spatial Precision and Microsecond Temporal Resolution, Biophys. J. 98, 2014 (2010).

[4] Y. Gu et al.: Three-Dimensional Super-Localization and Tracking of Single Gold Nanoparticles in Cells, Anal. Chem. 84, 4111 (2012).

[5] J. Ortega-Arroyo and P. Kukura: Interferometric Scattering Microscopy (ISCAT): New Frontiers in Ultrafast and Ultrasensitive Optical Microscopy, Phys. Chem. Chem. Phys. 14, 15625 (2012).

[6] D. Lasne et al.: Single Nanoparticle Photothermal Tracking (SNAPT) of 5-nm Gold Beads in Live Cells, Biophys. J. 91, 4598 (2006).

[7] C. Pache et al.: Fast Three-Dimensional Imaging of Gold Nanoparticles in Living Cells with Photothermal Optical Lock-in Optical Coherence Microscopy, Opt. Express 20, 21385 (2012).

[8] D. Lasne et al.: Label-Free Optical Imaging of Mitochondria in Live Cells, Opt. Express 15, 14184 (2007).

[9] F. Masia et al.: Resonant four-wave mixing of gold nanoparticles for three-dimensional cell microscopy, Optics Letters 34, 1816 (2009).

[10] F. Masia, W. Langbein, and P. Borri: Measurement of the Dynamics of Plasmons Inside Individual Gold Nanoparticles Using a Femtosecond Phase-Resolved Microscope, Phys. Rev. B 85, 235403 (2012).

[11] F. Masia, W. Langbein, and P. Borri: Polarization-resolved ultrafast dynamics of the complex polarizability in single gold nanoparticles, Phys.Chem. Chem. Phys. 15, 4226 (2013).

[12] George Zoriniants et al.: Background-Free 3D Nanometric Localization and Sub-nm Asymmetry Detection of Single Plasmonic Nanoparticles by Four-Wave Mixing Interferometry with Optical Vortices, Phys. Rev. $X 7,041022$ (2017). 